\title{
Pelvic Lymphadenectomy in the Treatment of Invasive Bladder Cancer: Literature Review
}

\author{
Ehab A. Elzayat ${ }^{1}$ and Ali A. Al-Zahrani ${ }^{2}$ \\ ${ }^{1}$ Department of Urology, Al-Azhar University, Cairo, Egypt \\ ${ }^{2}$ Department of Urology, University of Dammam, Saudi Arabia
}

Correspondence should be addressed to Ehab A. Elzayat, elzayat2003@yahoo.com

Received 1 January 2011; Accepted 15 May 2011

Academic Editor: Darius J. Bagli

Copyright $\odot 2011$ E. A. Elzayat and A. A. Al-Zahrani. This is an open access article distributed under the Creative Commons Attribution License, which permits unrestricted use, distribution, and reproduction in any medium, provided the original work is properly cited.

\begin{abstract}
The standard surgical treatment of invasive bladder cancer is the radical cystectomy and pelvic lymph node dissection (PLND). Up to one-third of patients with invasive bladder cancer have lymph node metastasis. Thus, PLND has important therapeutic and prognostic benefits. The number of lymph nodes that should be removed and the extent of the PLND are still a controversial issue. Recently, the trend of PLND increased toward more extended PLND. Several prognostic factors related to PLND were reported in the literature. In this paper, we will discuss the different PLND templates, number of lymph nodes that should be resected, lymph node density, lymphovascular invasion, tumor burden, extracapsular extension, and the aggregate lymph node metastasis diameter.
\end{abstract}

\section{Introduction}

According to cancer statistic 2010, bladder cancer is the fourth most common tumor in men in the United States, the number of new cases diagnosed were estimated to be 70,530 (52,760 men and 17,770 women), leading to 14,680 deaths [1]. In Europe, bladder cancer represents $6.6 \%$ and $2.1 \%$ of the total cancers and $4.1 \%$ and $1.8 \%$ of total deaths for cancer in men and women, respectively [2].

Radical cystectomy (RC) accompanied by pelvic lymph node dissection (PLND) is still the gold standard surgical treatment for muscle invasive bladder cancer. In the past, the role of PLND was purely diagnostic to rule out lymph nodes (LN) metastasis; currently, PLND is considered an essential part of surgical treatment of bladder cancer. Fourteen to $30 \%$ of the patients of invasive bladder cancer have LN metastasis at the time of RC $[3,4]$. The incidence of LN metastasis increased with higher tumor stage. LN-positive disease was found in 5\% patients with superficial bladder tumors and $18 \%$ of patients with $\mathrm{P} 2$ tumor, $26 \%$ of patients with P3a disease, and $46 \%$ of patients with P3b, and $42 \%$ of patients with P4 tumors [3].
It is common knowledge that the patients with LN metastasis have a poor prognosis, Vieweg et al. [5] reported on 140 patients with LN positive, $25.7 \%$ were disease-free, and $15.7 \%$ surviving beyond 5 years. In another study, the 3year survival in patients who underwent RC and PLND with negative and positive $\mathrm{LN}$ was $78.3 \%$ and $37.8 \%$, respectively [6]. Thus, PLND is associated with favorable prognosis and better cancer control. Four decades ago, many urologists did not perform PLND with RC [7]. In 1950, Kerr and Colby noted that the local recurrence rate decreased after cystectomy combined with PLND [8].

In 1973, Dretler et al. [9] reported the value of inclusion of PLND during RC without increasing the morbidity and mortality. Skinner [10] suggested that PLND can cure some patients with metastatic disease, effectively controls pelvic disease and can make a difference in survival.

In 1981 Smith and Whitmore [11] reported on one of the first anatomical LN mapping studies in patients undergoing $\mathrm{RC}$ and they suggested the possible therapeutic effect of a systematic bilateral PLND as a major determinant of patient survival. Although there is consensus the PLND should be an integral part of cystectomy; the extension of PLND is not 
standardized and the number of LNs that should be removed has yet to be defined [12].

In this article we will review the therapeutic and prognostic value of PLND, the optimal surgical template, and the minimum number of nodes to be removed.

\section{Lymphatic Drainage of the Bladder and Templates of PLND}

The lymphatic drainage of the bladder consists of the visceral lymphatic plexus inside the submucosa and the muscular layer, the small intercalated lymph nodes located within the perivesical fat, pelvic collecting trunks which is medial to the iliac LNs, regional pelvic LNs, which include the external and internal iliac, and sacral LNs, lymphatic trunks from the regional pelvic LNs to the common iliac LNs [13]. The pelvic LNs are embedded in fat and difficult to be appreciated during the surgery. The primary drainage sites include external and internal iliac and obturator LNs, secondary drainage from the common iliac LNs, and tertiary drainage from the trigone and posterior bladder wall is to the presacral nodes [14]. LNs mapping studies in RC shows that the rate of positive LNs detected decreased gradually from distal to more proximal sites and the most common site of LN metastasis were in the obturator and iliac LNs. Positive LNs were found in the perivesical fat and in the pelvic region in $22.7 \%$ of all patients, in the common iliac nodes in $8 \%$, in the presacral region in $5.1 \%$ and at or above the aortic bifurcation in 4\% [14]. In another study, the distribution of the LN metastasis in the external iliac, obturator, and internal iliac region was 33\%, 38\%, and 29\%, respectively. Metastases in only one region were found in $33 \%$ of patients $13 \%$ in the external iliac LNs, $10 \%$ in the obturator LNs, and $10 \%$ in the internal iliac LNs); $50 \%$ of all patients had lymph node metastases in the internal iliac region [15].

Abol-Enein et al. [16] in pathoanatomical study of LN involvement in patients with bladder cancer, concluded that the internal iliac and obtur ator LNs (endopelvic region) is the sentinel region of the lymphatic drainage of bladder cancer and there are no skipped lesions or isolated nodal metastasis above the aortic bifurcation. Thus any metastasis outside the true pelvis occurred only in multinodal disease and it was always associated with involvement of the obturator and/or internal iliac nodes. Others reported that single positive nodes were located outside of the pelvis in $27 \%$ of patients [17]. The difference between these finding may be explained by the variation in the natural history of the disease [16].

Early RC series suggested that it was not necessary to include the aortocaval lymph nodes in PLND, as a part of the cystectomy $[13,18]$. Others suggested the importance of an extended PLND to include common iliac LNs to remove all potential LN metastases [11].

The limits of each surgical template of PLND are not clearly defined in the urology guidelines. Although the absolute boundaries of the PLND remain a subject of controversy, three categories of PLND are reported in the literature, limited, standard, and extended. The limited PLND drains part of the primary drainage and includes only the obturator and external iliac LNs [7].

The standard PLND was defined as a removal of all nodal tissue of primary and secondary lymphatic drainage of the bladder and encompasses the common iliac bifurcation proximally, the genitor-femoral nerve laterally, the circumflex caudal iliac vein and lymph node of Cloquet distally, and the internal iliac vessels posteriorly, including the obturator fossa. The nodes around the proximal half of the common iliac artery/aortic bifurcation are spared to avoid injury to the hypogastric nerves [19].

The boundaries of extended PLND are $1-2 \mathrm{~cm}$ above the aortic bifurcation and common iliac vessels proximally (others may extend the PLND up to the level of inferior mesenteric artery), the genitofemoral nerve laterally, the circumflex iliac vein and lymph node of Cloquet distally, the internal iliac vessels posteriorly, including the obturator fossa, the presciatic nodes bilaterally, and the presacral lymph nodes over the sacral promontory [20].

Leissner et al. [17] defined three different anatomical levels of metastasis: Level I, included in the standard template; Level II, including the aortic bifurcation; Level III, including the para-aortic and paracaval areas. The authors noted that, if there is a nodal metastasis at Level I, positive nodes were also found in $57 \%$ and $31 \%$ of cases at Levels II and III, respectively. If positive nodes were found at Level II, $35 \%$ of cases were positive at Level III. Positive nodes at Level III were found only if metastases were present in 9 or more nodes at Levels I and II.

\section{Current Practice of PLND}

In 2004, Herr et al. [21] reported on 1091 consecutive RC performed by 16 experienced surgeons from 4 institutions between 2000 and 2002. Surgeons performed a standard PLND in $67 \%$ of patients, extended PLND in $13 \%$ of patients, and for various reasons $20 \%$ had a limited $(9 \%)$ or no node dissection (11\%). In analysis of the Surveillance, Epidemiology and End Results (SEER) data of 3603 RC performed between 1992 and 2003, Hollenbeck et al. [22] divided the hospitals according to the node count during cystectomy, low (no patients with $\geq 10$ LNs removed), medium (up to $20 \%$ of patients with $\geq 10 \mathrm{LNs}$ removed), and high (greater than $20 \%$ of patients with $\geq 10 \mathrm{LNs}$ removed). The authors found that only $0-4$ nodes were retrieved in $88.9 \%$ and $52.8 \%$ of cases in the low and high node count hospitals, respectively. The percentages of patients who had $\geq 10$ LNs removed were $0 \%$ at low LN count hospitals, $12.7 \%$ at medium LN count hospitals, and $35.3 \%$ at high LN count hospitals. It seems that the majority of cystectomy patients had $\leq 4$ LNs removed irrespective of the hospital and optimal PLND is not commonly performed.

The possible reasons for no or limited PNLD during RC was reported by Koppie et al. [23] who found that patients with older age and higher comorbidities were less likely to have PLND, and when PLND was performed, fewer LNs were evaluated. In another analysis of SEER data, Hellenthal et al. [24] noted that the odds of undergoing PLND 
( 1 or more nodes) decreased nearly $20 \%$ per 10 -year age increase. Also the odds increased by a factor of 1.5 in the tumor stage TisN0M0 to T3N0M0 and decreased in stage T4N0M0. The same authors concluded that $21 \%$ of patients did not have any LNs sampled at radical cystectomy. This number decreased from $37 \%$ in 1988 to $16 \%$ in 2004. During this period the mean number of LNs removed increased by 2.6 nodes and the percentage of patients undergoing any form of lymph node dissection increased by an average of $19 \%$. As of $2004,84 \%$ of patients had at least $1 \mathrm{LN}$ (and a mean of 13 nodes) examined at cystectomy. Similarly, Koppie et al. [25] noticed that the number of removed LN was associated with year of surgery in their series. The mean number of LNs removed during 1990-1994, 1994-1999, and 2000-2004 was 7.5, 8.6, and 14.7, respectively.

\section{Therapeutic and Prognostic Value of PLND}

4.1. Extent and Number of Lymph Nodes Removed. Weingärtner et al. [26] assessed the adequate number of LNs to be removed for achievement of complete and accurate PLND. Standard PLND was performed on 30 human cadavers and 59 consecutive patients with clinically organ confined prostate cancer during radical retropubic prostatectomy. The mean number of LNs removed in the autopsy series and from patients with prostate cancer was $22.7 \pm 10.2$ and $20.5 \pm 6.6$, respectively, with many interindividual differences. The authors concluded that the threshold of approximately 20 nodes was sufficient. The average number of nodes removed during the standard and extended PLND was reported to be 13 (9-18) and 31.5 (14.7-50), respectively [7].

The number of LNs retrieved during PLND is quite variable and different cutoff number of LNs that should be dissected was suggested. So far there is no consensus available for the standard number of LNs that should be retrieved in patients with bladder cancer. The higher number of LNs retrieved may reflect more complete RC and PLND. Several studies showed that disease-free survival or recurrence-free survival improved with more number of nodes retrieved which was an independent prognostic factor even after adjusting for node status, surgical margin, and pT stage [2729]. Herr et al. [27] found that the survival rate in the node negative patients was improved with more number of nodes removed, which may be attributed to an improved staging, and possible removal of undetectable micrometastasis. The authors suggested PLND of at least 9 or more as a minimum standard provides individual prognostic information.

Others reported on decreased risk of death in patients who had 10-14 lymph nodes removed. Patients with less than $3 \mathrm{LNs}$ retrieved were at significantly higher risk of death from bladder cancer than those with greater than 3 [30]. Leissner et al. [31] found that the extended PLND of $\geq 16 \mathrm{LNs}$ correlated with a higher percentage of patients with documented nodal metastases. There was a significant correlation between the number of removed LNs and the tumor-free 5year survival in patients with pT1, pT2, or pT3 tumors.
Fang et al. [32] reported on 349 patients who underwent RC and PLND between March 2000 and February 2008. The authors established an institutional policy mandating at least 16 LNs be examined in March 2004. Of all, 147 and 202 patients underwent surgery before and after the policy was implemented, respectively. The median number of LNs examined increased from 15 in the period before policy implementation to 20 in the 4 years after. Survival rates increased from $41.5 \%$ in the 4 years before policy implementation to $72.3 \%$ in the 4 years after. Capitanio et al. [33] reported on a multicenter study to identify the probability of finding one or more positive LNs based on the number of LNs removed. The authors found that removing $45 \mathrm{LNs}$ yielded a $90 \%$ probability. However, removing either 15 or 25 LNs indicated probability of $50 \%$ and $75 \%$, respectively. They concluded that removing $25 \mathrm{LNs}$ might represent the lowest threshold for the extent of PLND at RC. Others reported that at least 23 nodes would need to be removed in order to identify $80 \%$ of positive nodes [34].

The number of LNs resected is a surrogate for the extent of dissection and the quality of RC. Thus it seems to be difficult to establish a minimum or threshold number of LNs that should be removed during PLND due to the lack of a standardized template of PLND. According to Koppie et al. [25] removing 10 LNs may represent a thorough LN cleanout from a limited LN template, or a relatively incomplete dissection of LNs from an extended LN template. The authors concluded that no evidence was found to support a minimum number of LNs sufficient for optimizing bladder cancer outcomes when a limited or extended PLND is performed during RC. The probability of survival continues to increase as the number of LNs retrieved increases. Also, the authors recommended more extended PLND at the time of RC.

Several studies demonstrated that extended PLND decrease local recurrence and improve cure rates when it compared to limited and standard PLND [31,35]. Dhar et al. [36] reported on a multicenter study comparing the recurrence patterns and survival of 658 patients who underwent $R C$ with either limited or extended PLND. The overall LN-positive rate was $13 \%$ and $26 \%$ for patients with limited and extended PLND, respectively. The 5-year recurrence-free survival was $77 \%$ for pT2N0, $57 \%$ for pT3N0, and 35\% for node-positive tumors in the extended PLND group versus $67 \%, 23 \%$, and $7 \%$, respectively, in the limited PLND group $(P<0.0001)$.

In another study, LN metastases were detected in $38 \%$ and $17 \%$ of the extended and limited dissection groups, respectively. There was no significant difference in survival or time to recurrence between the 2 groups. However, the multivariate analysis demonstrated significantly improved survival and time to recurrence in the patients with extended PLND [37].

In addition to its therapeutic benefit, extended PLND offers more accurate staging compared to a limited/standard PLND. Dangle et al. [34] found that limited PLND would have missed $25 \%$ of LN-positive patients whereas standard PLND would have missed $11 \%$ of LN positive cases. Others noted that limited and standard PLND would have missed 27 and $10 \%$ of LN metastases in patients with a single positive lymph node, respectively [17]. Seiler et al. [15] found that 
PLND that do not include the internal iliac region misses $26 \%$ of all pelvic lymph nodes, $29 \%$ of metastases, and understages a substantial number of patients as pN0 (10\%).

Although surgical cure is rare in patients with gross nodal metastasis (N2-3), the RC with extended PLND can provide cure in this group of patients. The 10-year disease-free survival was reported in $24 \%$ of patients with surgery alone and $76 \%$ of patients died of disease. Thirty-two percent of patients with T2 tumors survived versus $9.7 \%$ of patients with stage T3 tumors [38].

4.2. Tumor Burden. The number of positive nodes retrieved is indicative of the tumor burden and considered as independent from the number of nodes removed. The survival rate is directly correlated with number of LN metastasis. Several cut off number of positive LNs was reported. One of these studies showed that the survival of patients with positive nodes was significantly better if $\leq 4$ positive nodes were removed than if there were $>4$ positive nodes $(37 \%$ versus $13 \%$ ) [27]. Improved overall survival was shown in a study by Lerner et al. [39] when 5 or fewer positive nodes were detected (40\% versus 10\%). The mean 3-year survival for patients with $1,2-5$, and $>5$ positive LNs was $58.6 \%, 31.8 \%$, and $6.8 \%$, respectively [6]. Fleischmann et al. [40] found that the Overall 5-year survival was $35 \%$ and $17 \%$ in patients with $<6$ and $\geq 6$ positive $\mathrm{LN}$, respectively. Others showed that the 10-year recurrence-free survival was significantly better in patients with $\leq 8$ positive nodes than in those with $>8$ metastatic nodes (40\% versus 10\%) [41]. Kassouf et al. [42] reported that the number of positive nodes was significantly associated with recurrence-free survival on univariate analysis $(P=0.04)$, but lost statistical significance on multivariable model $(P=0.055)$.

4.3. Lymph Node Density. LN density defined as number of positive LN divided by the total number of nodes removed and examined. It included 2 prognostic factors the tumor burden and the extent of PLND. The most commonly utilized LN density cut-point was $20 \%$. The concept of LN density was introduced and named "ratio-based" lymph node staging by Herr in 2003 [43]. They found that the 5-year overall survival decreased from $64 \%$ when the ratio was $\leq 20 \%$ down to $8 \%$ when it was $>20 \%$. The same concept was later reported under a different name (LN density) by Stein et al. [41] who showed decreased 10-year recurrencefree survival from $43 \%$ when the LN density was $\leq 20 \%$ to $17 \%$ when it was $>20 \%$. Others reported that the LN density showed some predictive ability, especially at a cutoff of $50 \%$ [30].

Kassouf et al. [44] compared nodal status and LN density in a multivariate model. For powerful LN density, a minimum number of 9 nodes need to be resected. The authors found that only LN density $>20 \%$ predicted decreased disease-specific survival and remained prognostic in patients who received adjuvant chemotherapy. Also the LN density is superior to tumor-node-metastasis (TNM) classification for nodal status in predicting disease-specific for susvii patients with LN-positive disease. This study and others support the use of LN density in the pathologic staging of node-positive bladder cancer [45].

Although Abdel-Latif et al. [6] found that both number of positive nodes ( 1 versus $2-5$ versus $>5$ ) and LN density ( $<10$ versus $10-20$ versus $>20 \%$ ) showed statistical significance on univariate analyses, only the number of positive nodes remained significant on multivariate modeling. Wright et al. [28] found statistically significant correlation between the number of positive nodes ( 1 versus 2 versus 3 versus $>3$ ) and lymph node density, and disease-specific and overall survival.

4.4. Lymphovascular Invasion. Lymphovascular invasion (LVI) means the presence of tumor cells in the endotheliumlined space. In a recent longitudinal evaluation of the prognostic value of LVI, Resnick et al. [46] found that $12.3 \%$ of patients had LVI at transurethral resection of the bladder tumour (TURBT) compared to $33.1 \%$ at RC. The risk of nodal disease was higher in those patients with LVI at TURBT than in those with no evidence of LVI at TURBT $(48.3 \%$ versus $25.0 \%, P<0.001)$. The authors concluded that the LVI has a useful prognostic value and should be incorporated into clinical decision making, particularly for RC in patients with superficial bladder carcinoma and the need for neoadjuvant chemotherapy.

Quek et al. [47] noted that the LVI is an important and independent prognostic variable in patients with invasive bladder cancer. It was significantly correlated with positive surgical margins, high pathological stages, older patients, and sex (female). Ten-year recurrence-free survival in patients without LVI was $74 \%$ compared with $42 \%$ in those with LVI $(P<0.0001)$. Similarly 10 -year overall survival was $43 \%$ in patients without LVI compared with $18 \%$ in those with LVI $(P<0.0001)$.

In another study, LVI was not significantly associated with age or sex, but was significantly associated with high pathological grade $(P=0.028)$, stage $(P<0.001)$, and node metastasis $(P<0.001)$. At the multivariate analysis, LVI was an independently significant prognostic factor for diseasespecific survival $[48,49]$. Others noted that the LVI in nodenegative patients is an adverse prognostic factor on univariate analysis of disease-specific survival, but not an independent prognostic factor on multivariate analysis [50].

Shariat et al. [51] reported on international validation of the prognostic value of LVI in 4257 patients treated with RC. In analysis, LVI was associated with both disease recurrence (hazard ratio $1.43, P<0.001$ ) and cancer-specific mortality $(1.45, P<0.001)$. In patients with negative LNs, LVI was independently associated with and improved the predictive accuracy of the standard predictors for recurrence (hazard ratio $1.68, P<0.001 ;+2.3 \%)$ and cancer-specific mortality (1.70, $P<0.001 ;+2.4 \%)$. The authors concluded that the LVI should be included in the staging of bladder cancer.

4.5. Extracapsular Extension of the Lymph Node (ECE). Currently, there are a few reports in the literature investigating the prognostic value of ECE of LN metastasis. Perforation and extension of the tumor growth outside the LN capsule 
indicate aggressive behavior of the tumor. Fleischmann et al. [40] found the ECE was observed in $58 \%$ of patients and in the multivariate analysis for recurrence-free survival, ECE of LN metastases was the strongest prognostic factor $(P=$ .019) of recurrence-free and overall survival. In contrast, Kassouf et al. [52] suggests that ECE is not an independent prognostic factor for overall survival, disease-specific survival, and recurrence-free survival in patients with positive LNs.

4.6. The Aggregate LN Metastasis Diameter (ALNMD). Some studies suggested that the size of the largest LN metastasis and/or the aggregate LN metastasis diameter (ALNMD) may provide prognostic information about the extent of LN metastasis, and the patients' survival. Mills et al. [53] noted that there is a significant association between the diameter of the largest LN metastasis and overall survival. Very recently, Stephenson et al. [54] reported on 134 positive LN patients treated with RC and minimum standard PLND. The median overall survival was 26 months for patients with ALNMD $\leq 20 \mathrm{~mm}$ versus 11 months for those with ALNMD $>20 \mathrm{~mm}$ $(P=.001)$. The authors concluded that ALNMD is a significant predictor of recurrence-free survival and overall survival and may provide a useful parameter to be included in the TNM-staging systems.

\section{Role of the Pathologist in LNs Assessment}

The accurate assessment of LN specimen depends on the carful work of the pathologist when searching the specimen for LNs and the way of specimen submission for pathological examination. Bochner et al. [55] found that individual LN specimen yielded more LNs compared to en bloc specimen in standard PLND (8.5 versus $2.4 \mathrm{LNs}, P=0.003$ ) and extended PLND (36.5 versus 22.6 LNs, $P=0.02$ ). This result confirmed by Stein et al. [56] who suggests 13 separate nodal packets to increase the total number of lymph nodes removed compared with en bloc submission.

The traditional method of detecting the LNs by sectioning and palpating the specimen may fail to detect the very small LNs. Koren et al. [57] described a new Lymph-node revealing solution (LNRS) for detecting LNs in PLND specimen. The solution comprised 95\% ethanol, diethyl ether, glacial acetic acid, and buffered formalin and used to degrease the tissue. The authors found that using the LNRS doubled the number of LNs yield, detected significantly smaller LNs, and improved nodal staging.

Herr et el. [58] reporting on pathologic evaluation of RC specimens, found that in $18 \%$ of patients, pathologists did not mention either the presence or the number of LNs. Standardized pathologic evaluation and reporting of RC and $\mathrm{LN}$ specimens is critical in cancer staging and design of clinical trials.

\section{Laparoscopic/Robot-Assisted PLND}

Laparoscopic PLND for prostate cancer was initially described by Schuessler et al. [59]. The laparoscopic surgery is minimally invasive with advantages of decreased blood loss, shorter hospital stay, and early recovery. Several reports showed that there is no significant difference in the intraoperative complications and the number of LNs removed by laparoscopic approach when compared with open surgery $[60,61]$.

Introduction of robot-assisted laparoscopic surgery added more field magnification with 3-dimensional vision and simulates the movements of the surgeon's wrist. Guru et al. [62] evaluated the number of LNs yield during robot-assisted $\mathrm{RC}$ and found that the mean operative time for PLND is 44 minutes (19-85) and the mean number of LNs removed was 18 (6-43).

It seems likely that the robot-assisted PLND can produce comparable results to open surgery; however, more studies of the techniques and learning curve are still needed [63].

\section{Morbidity and Mortality of PLND}

Although, RC is major surgery with potential high rates of complications, extended PLND does not increas morbidity or mortality. There is no significant difference between LNpositive and -negative patients in terms of postoperative complications [41]. In a study comparing extended PLND (up to the aortic bifurcation) to a more limited PLND, similar mortality rates were observed in the 2 groups [35]. Similarly, Leissner et al. [31] observed that the postoperative complications such as lymphocele and lymphoedema were similar in patients with $<16$ lymph nodes removed and patients with $>16$ nodes removed ( $2 \%$ versus $1 \%)$. Although the extended PLND increased the operative duration by 63 minutes, the limited and extended PLND patients did not differ significantly in terms of perioperative mortality and morbidity. Complications requiring surgical interventions occurred in 9\% patients in limited PLND and 11\% in extended PLND group $(P=0.28)$ [64].

\section{Conclusion}

PLND is an essential part of the surgical treatment of bladder cancer for its staging, curative, and prognostic role. The benefits of extended PLND were demonstrated in several studies with no significant difference in morbidity and mortality when compared to standard PLND. Despite the growing evidence that support the extended PLND up to the inferior mesenteric artery, the optimum PLND template is still controversial and its boundaries and the number of retrieved LNs have not yet been defined. Well-designed randomized controlled trials comparing standard to extended PLND in RC patients is still needed. The extent of PLND and the number of positive LNs are well-established risk factors; however, the cut off number of positive LNs is still to be defined. Several reports suggested that LVI and LN density should be included in the pathologic staging of bladder cancer. The prognostic value of ECE and ALNMD still need more investigations. 


\section{References}

[1] A. Jemal, R. Siegel, J. Xu, and E. Ward, "Cancer statistics, 2010," A Cancer Journal for Clinicians, vol. 60, no. 5, pp. 277$300,2010$.

[2] J. Ferlay, P. Autier, M. Boniol, M. Heanue, M. Colombet, and P. Boyle, "Estimates of the cancer incidence and mortality in Europe in 2006," Annals of Oncology, vol. 18, no. 3, pp. 581592, 2007.

[3] J. P. Stein, G. Lieskovsky, R. Cote et al., "Radical cystectomy in the treatment of invasive bladder cancer: long-term results in 1,054 patients," Journal of Clinical Oncology, vol. 19, no. 3, pp. 666-675, 2001.

[4] M. A. Ghoneim and H. Abol-Enein, "Lymphadenectomy with cystectomy: is it necessary and what is its extent?" European Urology, vol. 46, no. 4, pp. 457-461, 2004.

[5] J. Vieweg, W. F. Whitmore Jr., H. W. Herr et al., "The role of pelvic lymphadenectomy and radical cystectomy for lymph node positive bladder cancer. The memorial sloan-kettering cancer center experience," Cancer, vol. 73, no. 12, pp. 30203028, 1994.

[6] M. Abdel-Latif, H. Abol-Enein, M. El-Baz, and M. A. Ghoneim, "Nodal involvement in bladder cancer cases treated with radical cystectomy: incidence and prognosis," The Journal of Urology, vol. 172, no. 1, pp. 85-89, 2004.

[7] R. Hurle and R. Naspro, "Pelvic lymphadenectomy during radical cystectomy: a review of the literature," Surgical Oncology, vol. 19, pp. 208-220, 2010.

[8] W. S. Kerr and F. H. Colby, "Pelvic lymphadenectomy and total cystectomy in the treatment of carcinoma of the bladder," The Journal of Urology, vol. 63, pp. 842-851, 1950.

[9] S. Dretler, B. Ragsdale, and W. Leadbetter, "The value of pelvic lymphadenectomy in the surgical treatment of bladder cancer," The Journal of Urology, vol. 109, no. 3, pp. 414-416, 1973.

[10] D. G. Skinner, "Management of invasive bladder cancer: a meticulous pelvic node dissection can make a difference," The Journal of Urology, vol. 128, no. 1, pp. 34-36, 1982.

[11] J. A. Smith Jr. and W. F. Whitmore Jr., "Regional lymph node metastasis from bladder cancer," The Journal of Urology, vol. 126, pp. 591-593, 1981.

[12] A. Stenzl, N. C. Cowan, M. De Santis et al., "The updated EAU guidelines on muscle-invasive and metastatic bladder cancer," European Urology, vol. 55, no. 4, pp. 815-825, 2009.

[13] W. F. Leadbetter and J. F. Cooper, "Regional gland dissection for carcinoma of the bladder; a technique for one-stage cystectomy, gland dissection, and bilateral uretero-enterostomy," The Journal of Urology, vol. 63, no. 2, pp. 242-260, 1950.

[14] A. Vazina, D. Dugi, S. F. Shariat, J. Evans, R. Link, and S. P. Lerner, "Stage specific lymph node metastasis mapping in radical cystectomy specimens," The Journal of Urology, vol. 171, no. 5, pp. 1830-1834, 2004.

[15] R. Seiler, M. von Gunten, G. N. Thalmann, and A. Fleischmann, "Pelvic lymph nodes: distribution and nodal tumour burden of urothelial bladder cancer," Journal of Clinical Pathology, vol. 63, no. 6, pp. 504-507, 2010.

[16] H. Abol-Enein, M. El-Baz, M. A. Abd El-Hameed, M. AbdelLatif, and M. A. Ghoneim, "Lymph node involvement in patients with bladder cancer treated with radical cystectomy: a patho-anatomical study - a single center experience," The Journal of Urology, vol. 172, no. 5, part 1, pp. 1818-1821, 2004.

[17] J. Leissner, M. A. Ghoneim, H. Abol-Enein et al., "Extended radical lymphadenectomy in patients with urothelial bladder cancer: results of a prospective multicenter study," The Journal of Urology, vol. 171, no. 1, pp. 139-144, 2004.

[18] A. Ariyoshi, K. Minoda, K. Komatsu, Y. Fujisawa, A. Yamaguchi, and T. Yoshida, "Does 'extended' pelvic lymphadenectomy truly contribute to the management of bladder carcinoma?" European Urology, vol. 12, no. 5, pp. 314-317, 1986.

[19] R. D. Mills, A. Fleischmann, and U. E. Studer, "Radical cystectomy with an extended pelvic lymphadenectomy: rationale and results," Surgical Oncology Clinics of North America, vol. 16, no. 1, pp. 233-245, 2007.

[20] J. P. Stein, "Lymphadenectomy in bladder cancer: how high is "high enough"?" Urologic Oncology, vol. 24, no. 4, pp. 349355, 2006.

[21] H. Herr, C. Lee, S. Chang, and S. Lerner, "Standardization of radical cystectomy and pelvic lymph node dissection for bladder cancer: a collaborative group report," The Journal of Urology, vol. 171, no. 5, pp. 1823-1828, 2004.

[22] B. K. Hollenbeck, Z. Ye, S. L. Wong, J. E. Montie, and J. D. Birkmeyer, "Hospital lymph node counts and survival after radical cystectomy," Cancer, vol. 112, no. 4, pp. 806-812, 2008.

[23] T. M. Koppie, A. M. Serio, A. J. Vickers et al., "Age-adjusted Charlson comorbidity score is associated with treatment decisions and clinical outcomes for patients undergoing radical cystectomy for bladder cancer," Cancer, vol. 112, no. 11, pp. 2384-2392, 2008.

[24] N. J. Hellenthal, M. L. Ramírez, C. P. Evans, R. W. deVere White, and T. M. Koppie, "Trends in pelvic lymphadenectomy at the time of radical cystectomy: 1988 to 2004," The Journal of Urology, vol. 181, no. 6, pp. 2490-2495, 2009.

[25] T. M. Koppie, A. J. Vickers, K. Vora, G. Dalbagni, and B. H. Bochner, "Standardization of pelvic lymphadenectomy performed at radical cystectomy: can we establish a minimum number of lymph nodes that should be removed?" Cancer, vol. 107, no. 10, pp. 2368-2374, 2006.

[26] K. Weingärtner, A. Ramaswamy, A. Bittinger, E. W. Gerharz, D. Vöge, and H. Riedmiller, "Anatomical basis for pelvic lymphadenectomy in prostate cancer: results of an autopsy study and implications for the clinic," The Journal of Urology, vol. 156, no. 6, pp. 1969-1971, 1996.

[27] H. W. Herr, B. H. Bochner, G. Dalbagni, S. M. Donat, V. E. Reuter, and D. F. Bajorin, "Impact of the number of lymph nodes retrieved on outcome in patients with muscle invasive bladder cancer," The Journal of Urology, vol. 167, no. 3, pp. 1295-1298, 2002.

[28] J. L. Wright, D. W. Lin, and M. P. Porter, "The association between extent of lymphadenectomy and survival among patients with lymph node metastases undergoing radical cystectomy," Cancer, vol. 112, no. 11, pp. 2401-2408, 2008.

[29] B. R. Konety and S. A. Joslyn, "Factors influencing aggressive therapy for bladder cancer: an analysis of data from the SEER program," The Journal of Urology, vol. 170, no. 5, pp. 17651771, 2003.

[30] B. R. Konety, S. A. Joslyn, and M. A. O’Donnell, “Extent of pelvic lymphadenectomy and its impact on outcome in patients diagnosed with bladder cancer: analysis of data from the surveillance, epidemiology and end results program data base," The Journal of Urology, vol. 169, no. 3, pp. 946-950, 2003.

[31] J. Leissner, R. Hohenfellner, J. W. Thüroff, and H. K. Wolf, "Lymphadenectomy in patients with transitional cell carcinoma of the urinary bladder; significance for staging and prognosis," British Journal of Urology International, vol. 85, no. 7, pp. 817-823, 2000. 
[32] A. C. Fang, A. E. Ahmad, J. M. Whitson, L. D. Ferrell, P. R. Carroll, and B. R. Konety, "Effect of a minimum lymph node policy in radical cystectomy and pelvic lymphadenectomy on lymph node yields, lymph node positivity rates, lymph node density, and survivorship in patients with bladder cancer," Cancer, vol. 116, no. 8, pp. 1901-1908, 2010.

[33] U. Capitanio, N. Suardi, S. F. Shariat et al., "Assessing the minimum number of lymph nodes needed at radical cystectomy in patients with bladder cancer," British Journal of Urology International, vol. 103, no. 10, pp. 1359-1362, 2009.

[34] P. P. Dangle, M. C. Gong, R. R. Bahnson, and K. S. Pohar, "How do commonly performed lymphadenectomy templates influence bladder cancer nodal stage?" The Journal of Urology, vol. 183, no. 2, pp. 499-504, 2010.

[35] A. L. Poulsen, T. Horn, and K. Steven, "Radical cystectomy: extending the limits of pelvic lymph node dissection improves survival for patients with bladder cancer confined to the bladder wall," The Journal of Urology, vol. 160, no. 6, part 1, pp. 2015-2020, 1998.

[36] N. B. Dhar, S. C. Campbell, C. D. Zippe et al., "Outcomes in patients with urothelial carcinoma of the bladder with limited pelvic lymph node dissection," British Journal of Urology International, vol. 98, no. 6, pp. 1172-1175, 2006.

[37] M. Holmer, P. O. Bendahl, T. Davidsson, S. Gudjonsson, W. Månsson, and F. Liedberg, "Extended lymph node dissection in patients with urothelial cell carcinoma of the bladder: can it make a difference?" World Journal of Urology, vol. 27, no. 4, pp. 521-526, 2009.

[38] H. W. Herr and S. M. Donat, "Outcome of patients with grossly node positive bladder cancer after pelvic lymph node dissection and radical cystectomy," The Journal of Urology, vol. 165, no. 1, pp. 62-64, 2001.

[39] S. P. Lerner, D. G. Skinner, G. Lieskovsky et al., "The rationale for en bloc pelvic lymph node dissection for bladder cancer patients with nodal metastases: long-term results," The Journal of Urology, vol. 149, no. 4, pp. 758-764, 1993.

[40] A. Fleischmann, G. N. Thalmann, R. Markwalder, and U. E. Studer, "Extracapsular extension of pelvic lymph node metastases from urothelial carcinoma of the bladder is an independent prognostic factor," Journal of Clinical Oncology, vol. 23, no. 10, pp. 2358-2365, 2005.

[41] J. P. Stein, J. Cai, S. Groshen, and D. G. Skinner, "Risk factors for patients with pelvic lymph node metastases following radical cystectomy with en bloc pelvic lymphadenectomy: the concept of lymph node density," The Journal of Urology, vol. 170, no. 1, pp. 35-41, 2003.

[42] W. Kassouf, D. Leibovici, M. F. Munsell, C. P. Dinney, H. B. Grossman, and A. M. Kamat, "Evaluation of the relevance of lymph node density in a contemporary series of patients undergoing radical cystectomy," The Journal of Urology, vol. 176, no. 1, pp. 53-57, 2006.

[43] H. W. Herr, "Superiority of ratio based lymph node staging for bladder cancer," The Journal of Urology, vol. 169, no. 3, pp. 943-945, 2003.

[44] W. Kassouf, P. K. Agarwal, H. W. Herr et al., "Lymph node density is superior to TNM nodal status in predicting diseasespecific survival after radical cystectomy for bladder cancer: analysis of pooled data from MDACC and MSKCC," Journal of Clinical Oncology, vol. 26, no. 1, pp. 121-126, 2008.

[45] M. L. Quek and R. C. Flanigan, "The role of lymph node density in bladder cancer prognostication," World Journal of Urology, vol. 27, no. 1, pp. 27-32, 2009.

[46] M. J. Resnick, M. Bergey, L. Magerfleisch, J. E. Tomaszewski, S. B. Malkowicz, and T. J. Guzzo, "Longitudinal evaluation of the concordance and prognostic value of lymphovascular invasion in transurethral resection and radical cystectomy specimens," British Journal of Urology International, vol. 107, no. 1, pp. 4652, 2011.

[47] M. L. Quek, J. P. Stein, P. W. Nichols et al., "Prognostic significance of lymphovascular invasion of bladder cancer treated with radical cystectomy," The Journal of Urology, vol. 174, no. 1, pp. 103-106, 2005.

[48] F. Palmieri, E. Brunocilla, A. Bertaccini et al., "Prognostic value of lymphovascular invasion in bladder cancer in patients treated with radical cystectomy," Anticancer Research, vol. 30, pp. 2973-2976, 2010.

[49] C. Bolenz, E. Herrmann, P. J. Bastian et al., "Lymphovascular invasion is an independent predictor of oncological outcomes in patients with lymph node-negative urothelial bladder cancer treated by radical cystectomy: a multicentre validation trial," British Journal of Urology International, vol. 106, no. 4, pp. 493-499, 2010.

[50] M. Manoharan, D. Katkoori, T. A. Kishore, M. Jorda, T. Luongo, and M. S. Soloway, "Lymphovascular invasion in radical cystectomy specimen: is it an independent prognostic factor in patients without lymph node metastases?" World Journal of Urology, vol. 28, no. 2, pp. 233-237, 2010.

[51] S. F. Shariat, R. S. Svatek, D. Tilki et al., "International validation of the prognostic value of lymphovascular invasion in patients treated with radical cystectomy," British Journal of Urology International, vol. 105, no. 10, pp. 1402-1412, 2010.

[52] W. Kassouf, D. Leibovici, T. Luongo et al., "Relevance of extracapsular extension of pelvic lymph node metastases in patients with bladder cancer treated in the contemporary era," Cancer, vol. 107, no. 7, pp. 1491-1495, 2006.

[53] R. D. Mills, W. H. Turner, A. Fleischmann, R. Markwalder, G. N. Thalmann, and U. E. Studer, "Pelvic lymph node metastases from bladder cancer: outcome in 83 patients after radical cystectomy and pelvic lymphadenectomy," The Journal of Urology, vol. 166, no. 1, pp. 19-23, 2001.

[54] A. J. Stephenson, M. C. Gong, S. C. Campbell, A. F. Fergany, and D. E. Hansel, "Aggregate lymph node metastasis diameter and survival after radical cystectomy for invasive bladder cancer," The Journal of Urology, vol. 75, no. 2, pp. 382-386, 2010.

[55] B. H. Bochner, H. W. Herr, and V. E. Reuter, "Impact of separate versus en bloc pelvic lymph node dissection on the number of lymph nodes retrieved in cystectomy specimens," The Journal of Urology, vol. 166, no. 6, pp. 2295-2296, 2001.

[56] J. P. Stein, D. F. Penson, J. Cai et al., "Radical cystectomy with extended lymphadenectomy: evaluating separate package versus en bloc submission for node positive bladder cancer," The Journal of Urology, vol. 177, no. 3, pp. 876-882, 2007.

[57] R. Koren, A. Paz, D Lask et al., "Lymph-node revealing solution: a new method for detecting minute lymph nodes in cystectomy specimens," British Journal of Urology International, vol. 80 , no. 1 , pp. 40-43, 1997.

[58] H. W. Herr, J. R. Faulkner, H. B. Grossman, and E. D. Crawford, "Pathologic evaluation of radical cystectomy specimens: a cooperative group report," Cancer, vol. 100, pp. 2470-2475, 2004.

[59] W. W. Schuessler, T. G. Vancaillie, H. Reich, and D. P. Griffith, "Transperitoneal endosurgical lymphadenectomy in patients with localized prostate cancer," The Journal of Urology, vol. 145, no. 5, pp. 988-991, 1991.

[60] F. Porpiglia, J. Renard, M. Billia et al., "Open versus laparoscopy-assisted radical cystectomy: results of a prospective study," Journal of Endourology, vol. 21, no. 3, pp. 325-329, 2007. 
[61] A. K. Hemal and S. B. Kolla, "Comparison of laparoscopic and open radical cystoprostatectomy for localized bladder cancer with 3-year oncological followup: a single surgeon experience," The Journal of Urology, vol. 178, no. 6, pp. 2340-2343, 2007.

[62] K. A. Guru, K. Sternberg, G. E. Wilding et al., "The lymph node yield during robot-assisted radical cystectomy," British Journal of Urology International, vol. 102, no. 2, pp. 231-234, 2008.

[63] M. C. Schumacher, M. N. Jonsson, and N. P. Wiklund, "Does extended lymphadenectomy preclude laparoscopic or robotassisted radical cystectomy in advanced bladder cancer?" Current Opinion in Urology, vol. 19, pp. 527-532, 2009.

[64] C. Brossner, A. Pycha, A. Toth, C. Mian, and W. Kuber, "Does extended lymphadenectomy increase the morbidity of radical cystectomy?" British Journal of Urology International, vol. 93, pp. 64-66, 2004. 


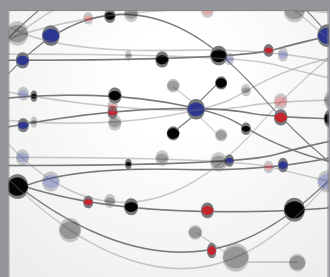

The Scientific World Journal
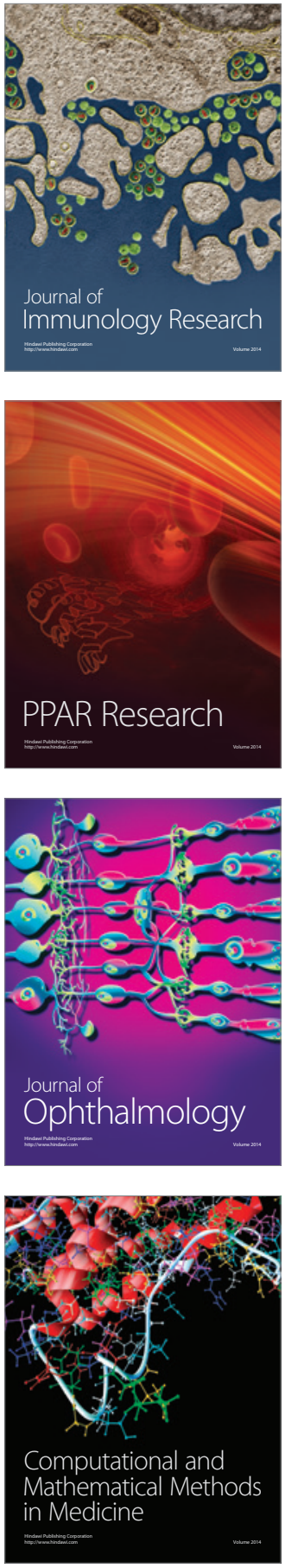

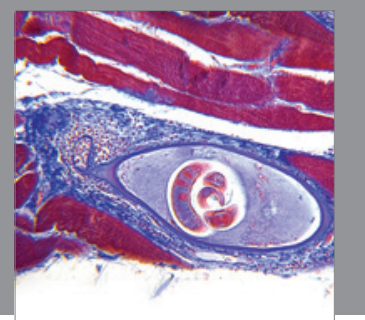

Gastroenterology

Research and Practice
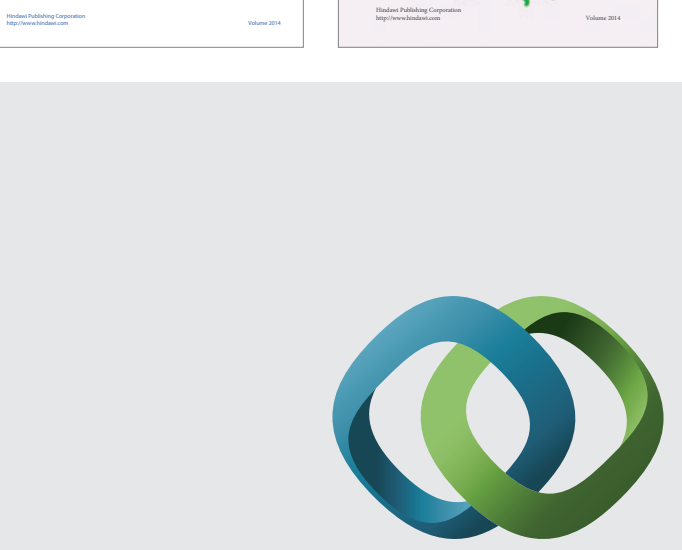

\section{Hindawi}

Submit your manuscripts at

http://www.hindawi.com
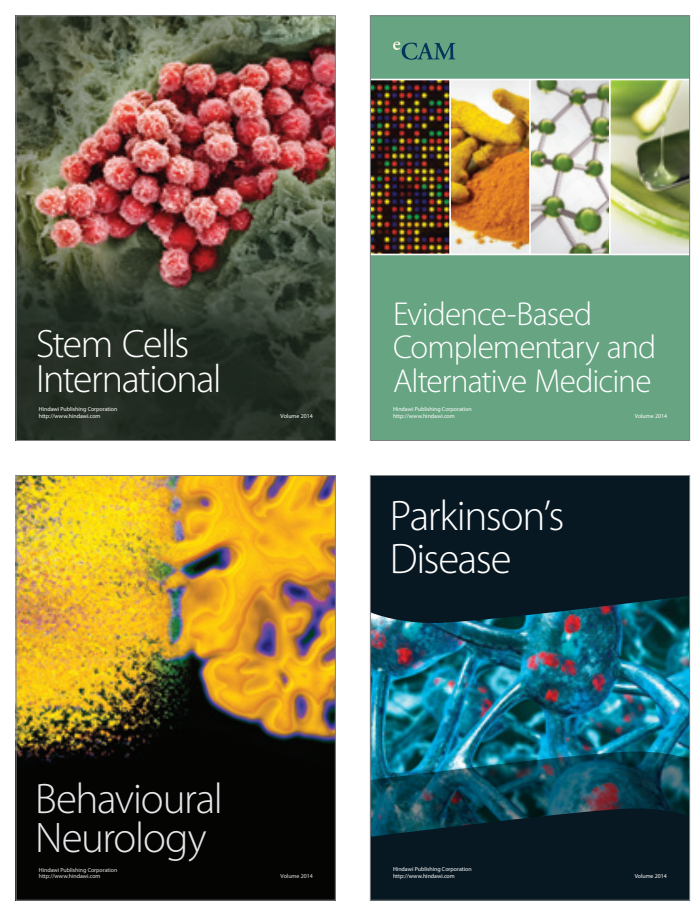

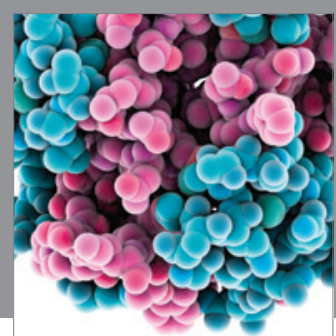

Journal of
Diabetes Research

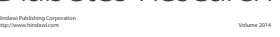

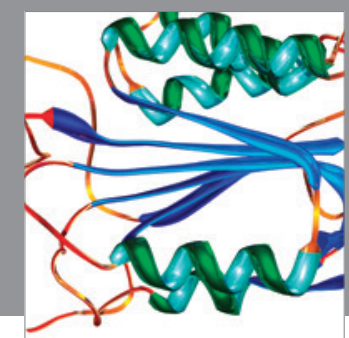

Disease Markers
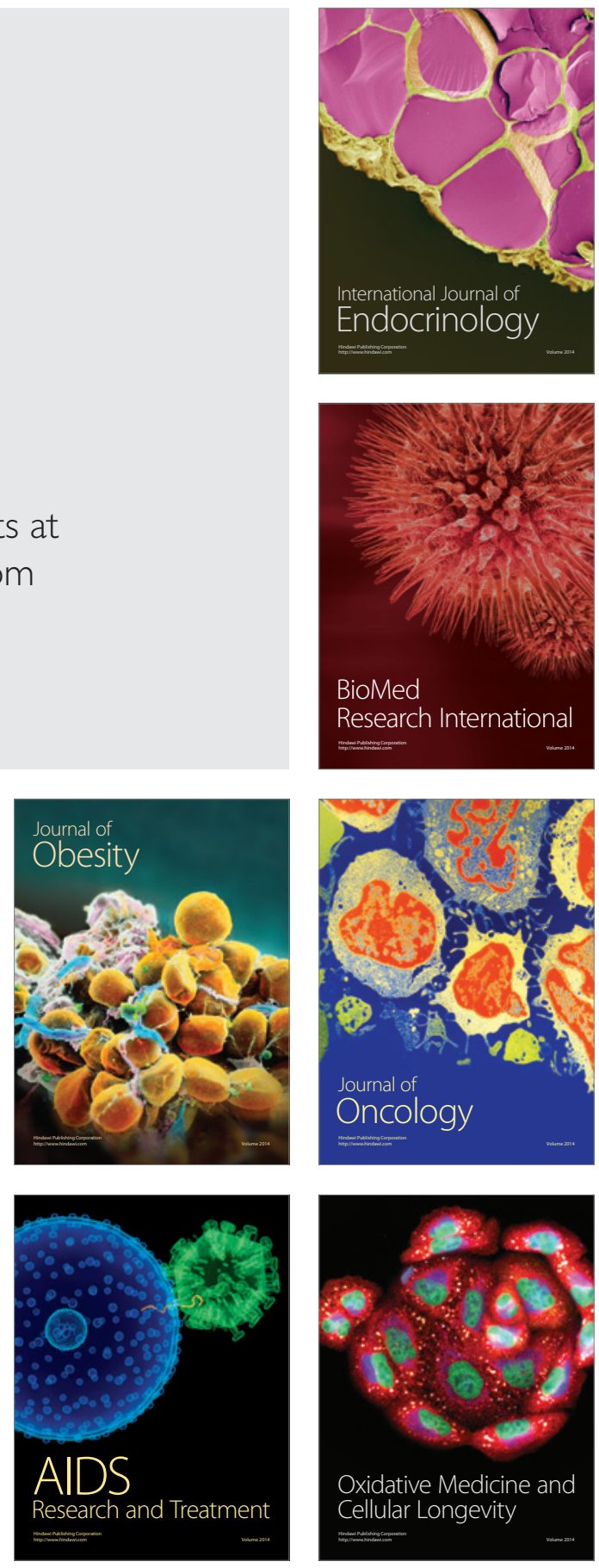